\title{
Le taux d'actualisation en Belgique
}

\author{
par Herbert Glejser*
}

\section{A. Les concepts et leur expression numérique}

En Belgique, l'analyse des coûts et avantages sociaux, y compris le recours à un taux d'actualisation, a été utilisée pour préparer des décisions d'investissement:

- dans le secteur public, en matière de priorité à des projets de voies routières (1) et de voies hydrauliques (2),

- dans le secteur privé (mais sous le contrôle des pouvoirs publics) en matière de choix entre centrales électriques classiques ou nucléaires.

Cette méthode d'analyse, toujours y compris un taux d'actualisation, a aussi servi à porter un jugement sur deux types de décision à caractère médical, à horizon lointain, mais ne comportant pas d'investissements physiques.

\section{B. Les apports et utilisations de capitaux}

1. Le Tableau 1 montre que les apports nets de capitaux proviennent, à concurrence de 500 milliards environ, des secteurs des particuliers ( $70 \%$ du total) et du reste du monde ( $30 \%$ du total). Le total de ces apports est utilisé dans une faible mesure par les entreprises privées $(5 \%)$ et les entreprises publiques $(9 \%)$, tandis que l'Etat absorbe $25 \%$ pour ses investissements et un peu plus de $60 \%$ pour la couverture de l'énorme déficit de ses opérations courantes.

2. La comptabilité nationale est une photographie statistique, mais n'implique aucune priorité et aucun processus permettant de dégager un taux d'actualisation. A cette fin, il nous faut encore procéder à des hypothèses simplificatrices en ce qui concerne:

- le secteur bénéficiaire du prêt net du reste du monde,

- la priorité de l'Etat et aux entreprises publiques sur l'utilisation de l'épargne des particuliers.

a) Le prêt net du reste du monde, soit 135 milliards, a été dirigé vers le secteur de l'Etat et vers les entreprises publiques à concurrence de 132 milliards; ceci n'a donc laissé que $135-132=3$ milliards comme utilisation nette de capitaux par le secteur privé

* Professeur aux Facultés Universitaires Notre-Dame de la Paix, Namur. 
Tableau 1

Les operations en capital des secteurs de l'èconomie belge en 1982 (milliards de francs)

\begin{tabular}{|c|c|c|c|c|c|}
\hline Secteur & \multicolumn{2}{|l|}{ Besoins de financement } & \multicolumn{3}{|l|}{ Moyens de financement } \\
\hline $\begin{array}{l}\text { Etat et entreprises } \\
\text { publiques }\end{array}$ & $\begin{array}{l}\text { Deficit courant de l'Etat }{ }^{1} \\
\text { Formation brute de capital } \\
\quad \text { fixe: Etat }{ }^{1} \\
\quad \begin{array}{l}\text { Entreprises } \\
\text { publiques }^{2}\end{array} \\
\end{array}$ & $\begin{array}{l}207 \\
502\end{array}$ & $\begin{array}{l}\text { Moyens propres } \\
\text { (amortissements) } \\
\text { Etat }{ }^{1} \\
\text { Entreprises } \\
\text { publiques }^{2} \\
\text { Epargne } \\
\text { de l'etranger } \\
\text { (hyp. 1) } \\
\text { des particuliers } \\
\text { (hyp. 2) } \\
\text { Total des moyens }\end{array}$ & $\begin{array}{l}135 \\
318 \\
318\end{array}$ & $\begin{array}{l}\frac{453}{502} \\
\end{array}$ \\
\hline Entreprises privees & $\begin{array}{l}\text { Formation brute de capital } \\
\text { fixe }^{2} \\
\text { Accroissement de stocks }^{1} \\
\text { Besoins totaux }\end{array}$ & $\begin{array}{r}333 \\
-12 \\
321\end{array}$ & $\begin{array}{l}\text { Moyens propres } \\
\quad \text { Amortissements }^{2} \\
\text { Benefices reserves }^{1} \\
\text { Epargne des particulie } \\
\text { Total des moyens }\end{array}$ & $\begin{array}{r}264 \\
29 \\
\mathrm{rs}^{6}\end{array}$ & $\begin{array}{r}293 \\
28 \\
321\end{array}$ \\
\hline Particuliers & $\begin{array}{l}\text { Construction d'habitation }{ }^{1} \\
\text { Consommation à credit }{ }^{3} \\
\text { Besoins totaux }\end{array}$ & $\begin{array}{r}135 \\
13 \\
148\end{array}$ & $\begin{array}{l}\text { Amortissement des } \\
\text { habitations }{ }^{2} \\
\text { Epargne des particulie } \\
\text { Moyens totaux }\end{array}$ & & $\begin{array}{r}49 \\
99 \\
148\end{array}$ \\
\hline Reste du monde & $\begin{array}{l}\text { Financement de l'Etat et } \\
\text { des entreprises publiques }\end{array}$ & 135 & $\begin{array}{l}\text { Prêt net du reste } \\
\text { du monde }\end{array}$ & & 135 \\
\hline
\end{tabular}

1 Comptabilitè nationale de 1982, chiffres rèvisès en 1983.

${ }^{2}$ Postes de la comptabilite nationale ventiles par nous en proportion de chiffres du tableau d'input-output de 1975: amortissements (entre habitations, entreprises privees, entreprises publiques et Etat); formation brute de capital fixe (idem). Le rapport des amortissements aux investissements dans les habitations ( est faible à cause de la longue duree de vie de celles-ci (60 ans).

3 Travaux de Pollefliet.

4 Il existe probablement une èpargne des entreprises publiques, mais pour l'instant elle est confondue avec celle de l'Etat.

${ }^{5}$ Ce chiffre a étè ventilè entre secteurs à partir de la balance des paiements de l'UEBL (dont nous avons soustrait certains flux relatifs au Luxembourg) ètablie par la Banque Nationale. Il tient compte des transferts nets en capital ( 5 milliards de la Belgique vers le reste du monde).

${ }^{6}$ Calcul par solde: $321-293=28$. 
(entreprises et particuliers). Dans un but de simplicité, nous supposerons que l'entièreté du prêt net du reste du monde a servi aux besoins en capitaux du secteur public.

b) L'Etat (pouvoir central régions et collectivités locales) et ses entreprises publiques seront censés bénéficier d'une priorité de fait sur l'épargne des particuliers. En effet, ce sont les Conseils des Ministres, de l'Etat central et accessoirement ceux des régions qui, en dernière analyse, fixent le montant total des investissements publics et sa répartition. Ce total dépend d'un quasi-objectif assez récent, limitant le rapport de l'endettement annuel nouveau à celui du produit national brut. Ce rapport, à son tour, est influencé par des objectifs d'équilibre de la balance des paiements (courante et en capitaux) et de stabilité des prix bien plus que par ceux de croissance et de plein emploi. Les autres objectifs de la politique économique (répartition des revenus, protection de branches par exemple) n'ont avec les investissements publics que des rapports plus lointains.

3. Moyennant ces deux hypothèses simplificatrices, nous aboutissons au Tableau 1 dans lequel les besoins de financement du secteur public sont couverts, tandis que les secteurs des entreprises privées et des particuliers se partagent ce qui reste de moyens de financement propres à ces deux secteurs (amortissements des entreprises privées et des habitations des particuliers, ainsi que bénéfices réservés des entreprises privées), plus 127 millions d'épargne des particuliers dont 28 vont aux entreprises privées et 99 demeurent dans le secteur des particuliers.

4. Les entreprises privées sont censées consacrer la totalité de leurs bénéfices réservés à leur autofinancement; en d'autres termes, il y a équivalence entre ce qu'elles pourraient prêter à l'Etat ou à certains particuliers et un supplément d'épargne qu'elles recevraient des particuliers, au-delà des 28 milliards du Tableau 1.

5. Reste le secteur des particuliers (3,5 millions de ménages) qui intervient dans notre raisonnement

- par 135 milliards d'investissements dans la construction d'habitations (dont 49 financés par leurs amortissements), encouragés par l'Etat pour des raisons politiques et sociales;

- par 13 milliards d'achats à crédit financés commercialement par des ventes et prêts à tempérament, des crédits bancaires en comptes courants et des cartes de crédit, et consistant surtout en biens durables (automobiles, électroménagers, télévisions); ces achats sont parfois découragés par l'Etat pour des considérations de stabilité des prix;

- last but not least, par le choix qu'ils font entre leur consommation privée ou une réduction partielle de celle-ci en vue d'obtenir des satisfactions futures grâce aux fruits de l'accroissement de leur épargne.

Si la consommation privée financée par les ressources courantes des particuliers n'apparaît pas directement au Tableau 1, elle y est sous-jacente à travers l'épargne des particuliers, qui est la seule concurrente de leur consommation pour l'utilisation de leur revenu disponible. On se rappellera que c'est la consommation privée, immédiate ou différée, qui est le point de départ de notre raisonnement sur le taux d'actualisation et que c'est l'appât 
de l'intérêt qui joue le rôle le plus important dans la détermination du taux d'actualisation.

\section{B. Le calcul du taux d'actualisation}

1. Tout apport de capitaux utilisés par le secteur public a un coût d'opportunité, car il n'est pas disponible pour d'autres usages. Nous classons ces derniers en quatre catégories (voir les colonnes du Tableau 2):

- les investissements des entreprises privées

- les investissements des particuliers en habitations

- la consommation privée avec recours au crédit

- la consommation privée sans recours au crédit.

Ce raisonnement s'apparente à celui de Harberger (11), qui envisage, lui aussi, une moyenne pondérée de divers taux applicables à des investissements déplacés mais il s'agit, pour lui, de taux marginaux de productivité du capital (pour les utilisations de capitaux) et des taux marginaux de préférence pour le présent (pour les apports de capitaux stimulés par la hausse du taux d'intérêt qui résultera de l'investissement public envisagé). Malheureusement, cet auteur ne va pas jusqu'à chiffrer les résultats de la méthode qu'il préconise.

2. Le coût social de chaque usage alternatif, exprimé en \% sous la forme d'un taux d'intérêt réel, s'obtient en corrigeant le taux des emprunts publics pour quatre raisons (voir les lignes du Tableau 2):

- l'intermédiation;

- les subventions;

- les impôts directs sur les revenus des particuliers;

- les exonérations d'impôts directs sur les revenus des particuliers.

Le point de départ de notre raisonnement est le taux des emprunts publics, dont l'importance relative est nettement dominante. Nous avons estimé la durée moyenne des emprunts conclus en vue d'usages alternatifs:

- pour les investissements des entreprises privées, à 8 ans;

- pour les investissements des particuliers en logements, à 8 ans;

- pour la consommation privée avec recours au crédit, à 2 ans;

- pour la consommation privée sans recours au crédit, à 5 ans (la durée moyenne des emprunts publics dans lesquels se place l'épargne des particuliers).

En octobre 1984, le taux d'intérêt des emprunts publics (rendement à l'échéance) se montait à

$10,0 \%$ pour une échéance à 2 ans;

$11,1 \%$ pour une échéance à 5 ans;

$11,75 \%$ pour une échéance à 8 ans; 
l'inflation était de 5,3\% par an et l'illusion monétaire négligeable. Ceci donne des taux réels de $4,6 \%, 5,6 \%$ et $6,1 \%$ que nous avons acceptés pour $1982\left(^{*}\right)$.

Les taux nominaux des emprunts publics émis en 1982 à ces diverses échéances sont connus, mais le passage aux taux réels est difficile parce qu'à cette époque le taux d'inflation anticipé était probablement supérieur à ceux qui ont pu être effectivement constatés ex post. En effet, peu avant la dévaluation du franc belge en février 1982, la Banque Nationale de Belgique avait affirmé qu'une hausse des monnaies étrangères provoquerait, au bout de quelques mois, une hausse double de l'index des prix à la consommation - ce qui ne s'est pas produit, notamment par suite de la modération des revenus imposée par le gouvernement.

Devant cette situation, nous avons supposé que les taux réels (inflation déduite) de 1982 étaient les mêmes que ceux de 1984, année pour laquelle l'inflation ex post de 5,3\% peut être considérée comme très proche du taux prévu par les conjoncturistes tenant justement compte des conséquences des déficits budgétaires américains et belges.

3. Les risques que prennent ceux qui achètent des fonds d'Etat belges ne sont pas nuls, car il peut arriver que des dettes de l'Etat ne soient pas remboursées, ou soient remboursées en francs dépréciés beaucoup plus rapidement que ce que le marché prévoyait lors de l'émission des obligations, ou encore que les emprunts fassent l'objet d'une conversion forcée réduisant le taux d'intérêt convenu. Cependant, nos calculs ne tiennent pas compte de ces risques.

Notons aussi que le coût réel pour l'Etat de ses emprunts est plus élevé que les chiffres qui précèdent, car il doit payer pour l'émission une intermédiation financière de $2 \%$ à répartir sur une durée moyenne de 8 ans, soit $0,25 \%$ par an.

4. Les subventions sont accordées par l'Etat et par les autorités régionales, certainement de manière excessive, pour des raisons de concurrence internationale, de politique régionale et accessoirement de plein emploi national. Elles prennent parfois les formes de prêts publics à taux réduit, ou de garanties données à certains emprunts; les montants ainsi octroyés apparaissent soit dans les dépenses courantes de l'Etat et des régions, soit dans leurs dépenses en capital de l'année, soit dans les dépenses de capital d'une année ultérieure (lorsqu'un prêteur privé fait appel aux garants) ou parfois n'apparaissent pas du tout (lorsque l'Etat n'insiste pas pour obtenir le paiement des impôts et des cotisations de sécurité sociale et que l'entreprise privée ne peut faire face à ses engagements).

5. Les lignes (1) à (7) du Tableau 2, donnant le détail des calculs conduisent, à la ligne (8), aux taux d'escompte sociaux associés aux usages alternatifs soit (en termes réels, inflation déduite):

- investissements privés:

- des entreprises $\quad 7,8 \%$

- des particuliers (logements) $\quad 3,2 \%$

* On notera que lors de la dernière période sans inflation, les emprunts publics, censés ne présenter aucun risque, donnaient un taux d'intérêt de $4,4 \%$; nous n'avons pas utilisé ce chiffre, parce qu'il date déjà d'une trentaine d'années (1951 à 1955). 
Tableau 2

Le calcul du taux d'actualisation à partir des usages alternatifs évincés par les emprunts du secteur public belge en 1982

\begin{tabular}{|c|c|c|c|c|}
\hline & \multicolumn{2}{|c|}{ Investissements privés } & \multicolumn{2}{|c|}{ Consommation privée } \\
\hline & $\begin{array}{l}\text { des entre- } \\
\text { prises }\end{array}$ & $\begin{array}{l}\text { des parti- } \\
\text { culiers } \\
\text { (logement) }\end{array}$ & $\begin{array}{l}\text { avec recours } \\
\text { au crédit }\end{array}$ & $\begin{array}{l}\text { sans recours } \\
\text { au crédit }\end{array}$ \\
\hline Durée de l'emprunt & 8 ans & 8 ans & 2 ans & 5 ans \\
\hline $\begin{array}{l}\text { Taux d'intérêt payés par } \\
\text { l'Etat } \\
\text { (1) nominaux } \\
\text { (2) réels }{ }^{1}\end{array}$ & $\begin{array}{r}11,75 \% \\
6,1 \%\end{array}$ & $\begin{array}{r}11,75 \% \\
6,1 \%\end{array}$ & $\begin{array}{r}10,0 \% \\
4,5 \%\end{array}$ & $\begin{array}{r}11,1 \% \\
5,5 \%\end{array}$ \\
\hline $\begin{array}{l}\text { Corrections pour l'inter- } \\
\text { médiation: } \\
\text { Risque additionnel et frais } \\
\text { du prêteur } \\
\text { Commissions } \\
\text { Divers }\end{array}$ & $\begin{array}{l}+2,7 \% \\
+0,5 \%\end{array}$ & $\begin{array}{c}- \\
- \\
+0,4 \%\end{array}$ & $\begin{array}{c}+3,5 \% \\
- \\
+0,5 \%^{3}\end{array}$ & \\
\hline (3) Total & $+3,2 \%$ & $+0,4 \%$ & $+4,0 \%$ & \\
\hline (4) Pour les subventions & $-1,5 \%$ & $-0,9 \%$ & - & - \\
\hline $\begin{array}{l}\text { (5) Pour le précompte } \\
\text { sur les revenus mobiliers }\end{array}$ & - & - & - & $2,2 \% 4$ \\
\hline $\begin{array}{l}\text { (6) Pour l'exonération } \\
\text { d'impôts directs }\end{array}$ & - & $-2,4 \%{ }^{5}$ & - & - \\
\hline $\begin{array}{l}(7)=(3)+(4)+(5)+(6) \\
\text { Total des corrections }\end{array}$ & $+1,7 \%$ & $-2,9 \%$ & $+4,0 \%$ & $-2,2 \%$ \\
\hline $\begin{array}{l}(8)=(2)+(7) \\
\text { Taux d'escompte sociaux }\end{array}$ & $7,8 \%$ & $3,2 \%$ & $8,5 \%$ & $3,3 \%$ \\
\hline $\begin{array}{l}\text { (9) Montants susceptibles } \\
\text { d'être placés } \\
\text { (Tableau 2, milliards) }\end{array}$ & 333 & 135 & $13^{2}$ & 446 \\
\hline $\begin{array}{l}\text { (10) Coefficients de passage } \\
\text { des parts moyennes } \\
\text { aux parts marginales }\end{array}$ & 3 & 5 & 1 & 1 \\
\hline $\begin{array}{l}(11)=(9) \times(10) \\
\text { Coefficients }\end{array}$ & 999 & 675 & 13 & 446 \\
\hline
\end{tabular}




\begin{tabular}{|l|c|c|c|c|}
\hline \multirow{2}{*}{} & \multicolumn{2}{|c|}{ Investissements privés } & \multicolumn{2}{c|}{ Consommation privée } \\
\cline { 2 - 5 } & $\begin{array}{c}\text { des entre- } \\
\text { prises }\end{array}$ & $\begin{array}{c}\text { des parti- } \\
\text { culiers } \\
\text { (logement) }\end{array}$ & $\begin{array}{c}\text { avec recours } \\
\text { au crédit }\end{array}$ & $\begin{array}{c}\text { sans recours } \\
\text { au crédit }\end{array}$ \\
\hline$(12)$ Idem, total =1 & 0,469 & 0,316 & 0,006 & 0,209 \\
\hline $\begin{array}{l}(13)=(8) \times(12) \\
\text { Contribution au taux } \\
\text { d'actualisation }\end{array}$ & 3,66 & 1,01 & $0,05^{\circ}$ & 0,69 \\
\hline Moyenne pondérée & \multicolumn{4}{|c|}{$5,41 \%$ arrondi à $5,4 \%$} \\
\hline
\end{tabular}

${ }^{1}$ Calculés à partir du taux d'inflation de 1984 , soit $5,3 \%$; par exemple $\frac{1,1175}{1,0530}=1,061$

${ }^{2}$ Ces chiffres très faibles sont dus au fait que l'année 1982 a connu un freinage.

${ }^{3}$ Assurance-crédit.

4 Précompte de $20 \%$ sur $11,1 \%$ d'intérêt nominal.

${ }^{5}$ L'exonération porte sur $44 \%$ (taux d'impôt marginal des propriétaires d'immeubles) de $(6,1+0,4-0,9)$ ou $0,44 \times 5,6 \%=2,4 \%$.

${ }^{6}$ Frais d'expertise, de géomètre, de notaire ( $3 \%$ répartis sur 8 ans).

- consommation privée:

- avec recours au crédit

- sans recours au crédit

6. Nous avons envisagé d'estimer l'effet d'éviction en pondérant ces quatre taux par les parts respectives des usages alternatifs en 1982, qui ressortent du Tableau 1 et sont repris à la ligne (9) du Tableau 2. Cette méthode aurait conduit à un taux moyen pondéré de 5,0\%; elle souffre cependant du fait qu'elle implique que les parts marginales des usages alternatifs sont égales à leurs parts moyennes, ce qui est très improbable.

7. Malheureusement l'estimation des parts marginales des usages évincés présente de très grandes difficultés. Elle présuppose en effet, une spécification complète suivie d'une estimation économétrique des fonctions d'investissement des entreprises, d'investissements en logement par les particuliers, d'achats de biens de consommation durable par les particuliers et enfin de consommation privée, dans lesquelles le taux d'intérêt (dont l'effet seul nous intéresse ici) ne serait qu'une variable parmi d'autres; de la sorte, on pourrait évaluer l'élasticité des quatre composantes par rapport à ce taux d'intérêt. Encore faudrait-il que ce dernier soit exprimé en termes réels, c'est-à-dire corrigé d'une compensation pour la hausse des prix anticipée. Or celle-ci est extrêmement difficile à estimer. Pareille étude sortirait donc du cadre de cet exposé.

8. A considérer les statistiques d'évolution du taux d'intérêt et des quatre composantes qui nous occupent au cours de la période allant de 1968 à 1983, on constate pour le taux 
d'intérêt nominal, une hausse rapide de 1968 à 1971, suivie d'une augmentation très lente au cours de toute la décennie 1970 et d'une nouvelle et très forte accélération au cours des années quatre-vingt; sauf pendant la période de 1970 à 1979, la hausse des taux nominaux a été telle qu'on peut admettre que le taux réel a crû lui aussi; par contre, au cours des années soixante-dix, le taux réel a sans doute diminué.

Ce sont les investissements en immeubles d'habitation et, dans une moindre mesure, ceux des entreprises, qui révèlent apparemment la plus grande sensibilité à ces fluctuations du taux d'intérêt.

Enfin les achats de biens de consommation durables et la consommation privée dans son ensemble ne paraissent guère affectés.

Nous proposons dès lors les poids suivants pour chacune de ces composantes:

- Immeubles d'habitation:

- Investissements des entreprises:

- Consommation de biens durables:

- Consommation dans son ensemble:

Ces chiffres figurent à la ligne (10) du Tableau 2 .

Nos quatre élasticités marginales ont été multipliées par les montants - ligne (9) - des dépenses correspondantes en 1982, aboutissant ainsi aux coefficients de pondération des lignes (11) et (12) qu'il convient d'affecter aux quatre taux d'escompte sociaux.

Enfin, la contribution de chacun des quatre usages alternatifs au taux d'actualisation apparaît à la ligne (13) et le total de ces contributions est la moyenne pondérée recherchée des quatre taux d'escompte sociaux, c'est-à-dire le taux d'actualisation. Il s'élève à $5,41 \%$, chiffre que nous avons arrondi à $5,4 \%$. 